\title{
ANN-Based Modeling for Load and Main Steam Pressure Characteristics of a 600MW Supercritical Power Generating Unit
}

\author{
Liangyu Ma, Zhiyuan Gao \\ Automation Department, School of Control and Computer Engineering \\ North China Electric Power University, \\ Baoding, 071003, China \\ maliangyu@ncepu.edu.cn; 771749447@qq.com
}

\begin{abstract}
A supercritical coal-fired power generating unit is a typical multivariable system with large inertia and non-linear, slow time-variant, time-delay characteristics, which often makes the coordinated control quality deteriorate under widerange load-changing conditions, and can't well satisfy the unit load and main steam pressure control requirements. Thus, it is of vital significance to study the supercritical boiler unit's operation characteristic by means of modeling method, and to improve the control quality with model-based advanced control strategies. In this paper, artificial neural network (ANN) method was used to build a nonlinear mathematical model of the load and main steam pressure characteristics for a 600MW supercritical boiler unit. Operation data over widerange load-changing conditions were used for model training. Simulation tests showed that the model can fit the complex non-linear, dynamic characteristics between the unit's load, main steam pressure and fuel, feedwater flow and turbine governing valve opening with high precision and strong generalization ability. The model can be used as a prediction model to construct an intelligent controller for supercritical boiler unit coordinated control to meet the engineering application demand.
\end{abstract}

Keywords-supercritical power unit; nonlinear mathematical model; artificial neural network; coordinated control system

\section{INTRODUCTION}

Supercritical (SC) or ultra-supercritical (USC) coal-fired power units are increasingly becoming the main generator units of the power grid because of their high efficiency and environment-friendly characteristics, compared to subcritical coal-fired units. A SC/USC boiler unit is a multivariable system with large inertia and non-linear, slow time-variant characteristics, which brings many problems for its coordinated control system (CCS) design and optimization. It is of vital significance to build a mathematical model for a SC/USC boiler unit and to study the boiler unit's operation characteristics by means of modeling method, which helps to improve the coordination control quality with model-based advanced control strategies.

In recent years, along with wide application of the supercritical or ultra-supercritical boiler units, many scholars have developed various models for SC/USC boiler units [29]. However, it is still an important issue to establish a nonlinear mathematical model with high precision and strong usability, which is easy to be used in controllers' design and development for a once-through boiler unit.

With the rapid development of computer technology, many experts and scholars have put their attention to Artificial Neural Network (ANN) modeling method. A neural network model with strong non-linear mapping, adaptive learning, association memory ability and good fault tolerance can overcome huge computation and complex identification process of the traditional modeling method.

This paper presents a non-linear dynamic model for the load and main steam pressure characteristics of a $600 \mathrm{MW}$ supercritical once-through unit based on a non-linear autoregression with exogenous inputs (NARX) neural network model constructed with a traditional BP network. The model is validated by detailed simulation tests.

\section{BP NEURAL NETWORK PRINCIPLE}

\section{A. Neural Network Structure}

In this work, a typical BP neural network is applied to build the mathematical model for the load and steam pressure characteristics of a supercritical boiler unit.

BP network is a kind of forward multi-layer neural network. The network achieves minimum Mean Squared Error (MSE) by adjusting the weights and thresholds of the network continuously according to the error backpropagation algorithm. BP network is composed of the input layer, hidden layer(s) and output layer. The basic neuron and a typical three-layer neural network structure are shown in Fig. 1.

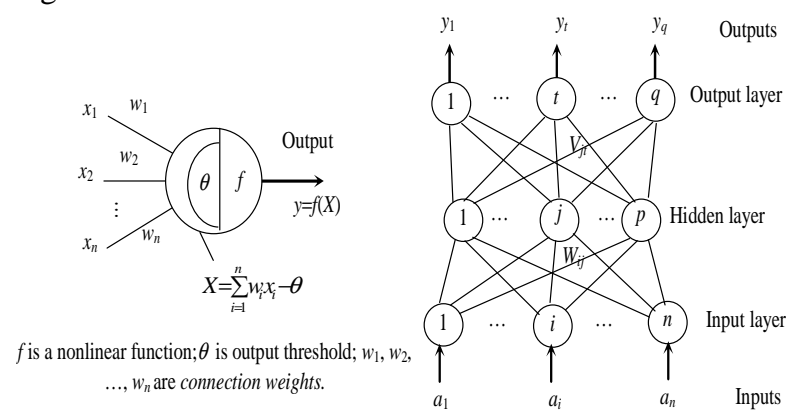

Figure 1. Neuron structure and a three-layer BP network. 


\section{B. Principle of BP Training Algorithm}

BP algorithm is mainly divided into two stages [10]: forward propagation stage and back propagation stage. In forward propagation stage, a sample $\left(\boldsymbol{X}_{\boldsymbol{P}}, \boldsymbol{Y}_{\boldsymbol{P}}\right)$ is taken from the sample set, and $\boldsymbol{X}_{\boldsymbol{P}}$ is used as the network's input to calculate the corresponding output $\boldsymbol{O}_{\boldsymbol{P}}$. In this stage, the information is transformed and transferred step by step from the input layer to the output layer. In the back propagation stage, the error between the calculated output $\boldsymbol{O}_{\boldsymbol{P}}$ and the ideal output $\boldsymbol{Y}_{\boldsymbol{P}}$ is calculated and the weight matrix is adjusted to minimize the error. Generally the back propagation phase is controlled by the error index.

The $P$ th sample's error measure of the network is defined as

$$
E_{P}=\frac{1}{2} \sum_{j=1}^{m}\left(Y_{P j}-O_{P j}\right)^{2}
$$

The error measure of the whole sample set is defined as

$$
E=\sum_{p=1}^{n} E_{P}=\frac{1}{2} \sum_{p=1}^{n} \sum_{j=1}^{m}\left(Y_{P j}-O_{P j}\right)^{2}
$$

The connection weights' adjustment process for each layer is going round and round along with information positive propagation and error back propagation, as far as the error is reduced to an acceptable level or the process achieves preset number of steps.

Based on the error back propagation principle, a lot of improved algorithms have been proposed to improve the network's convergence performance. Among them, LM algorithm (Levenberg-Marquardt method) is known as one of the most effective methods with fast convergence performance [11], which will be used for model development in this paper.

\section{NEURAL NETWORK MODELING FOR A 600MW SUPERCRITICAL BOILER UNIT}

\section{A. Model Variables Selection}

During the load increasing of a SC/USC boiler unit, the main steam will convert from wet state into dry state and finally into "once-through" stage. In this work, the modeling load scope is limited to the unit's once-through stage. In this stage, the SC/USC boiler unit's characteristics can be simplified into a model with three inputs and three outputs, which describes the nonlinear relationship between fuel flow, feedwater flow, turbine governing valve opening and unit's load, main steam pressure, intermediate point temperature (heat enthalpy). In view of the modeling purpose is for coordination control, which is only directly related to the unit's load and main steam pressure, we don't consider the intermediate point temperature (heat enthalpy) during modeling. Thus the properties of the coordinated control object can be simplified into a non-linear model with three inputs and two outputs, as shown in Fig. 2.

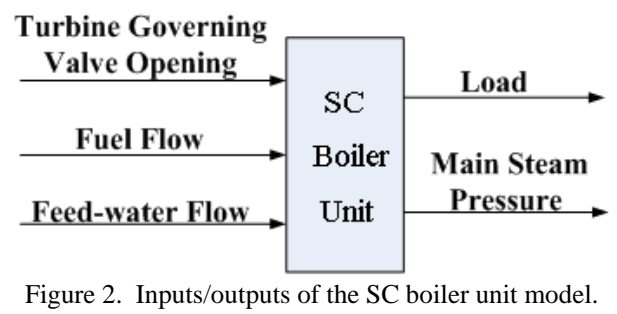

\section{B. Neural Network Model Structure}

Based on the basic BP neural network shown in Fig. 1, the NN structure in Fig. 3 is used to model the load and the steam pressure characteristics of the supercritical oncethrough boiler unit. This model includes one-order timedelay inputs and the outputs' time-delay feedback. It is essentially a simplified non-linear auto-regressive network with exogenous inputs (NARX) expressed by BP neural network, which considers the influence of the previous step's inputs and outputs on the current step's outputs.

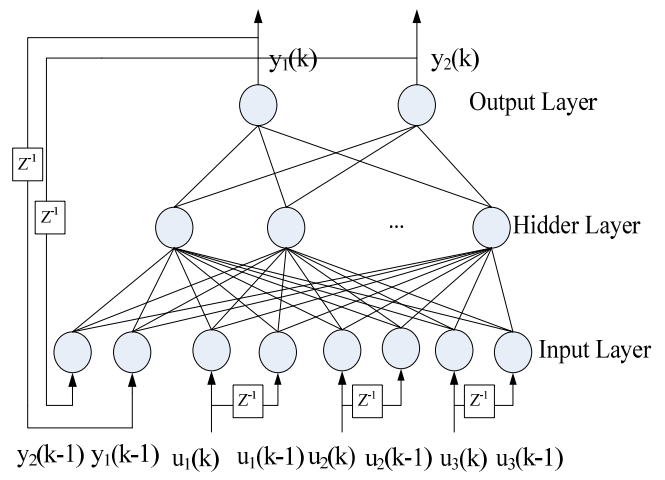

Figure 3. Simplified NARX neural network model.

For the model shown in Fig. 3, the network design is turned to optimal selection of the number of hidden layer neurons and determination of weights and thresholds after the inputs and outputs are determined.

In this paper, neural network models with different hidden-layer neurons are trained. The optimal system model is established by comparing the convergence performance of each model. For the above-mentioned network model, TANSIG function is chosen as the excitation function from input to hidden layer and PURELIN function is chosen as that from hidden to output layer.

\section{Training Data Preparation}

The neural network model should be trained with sample data after the model's inputs, outputs and structure are determined. To make the neural network model comprehensively reflect the dynamic and static characteristics of the controlled object, the training data should be wide enough to involve the different conditions, under which the model will be applied, containing different typical steady-state conditions and load-changing dynamic processes.

In this paper, the model's training sample data were got from the full-scope power plant simulator of a 600MW supercritical coal-fired power unit. During data acquisition 
process, the simulator was put in coordinated control mode, and feedwater control, all levels of superheated steam temperature control, air flow control, etc were all put into automatic modes. In our work, 23,854 sets of data were collected from the simulator with the sampling period of $1 \mathrm{~s}$, including steady-state data at 600MW, 540MW, 480MW, 420MW and 360MW load levels, and the dynamic transient data between the above five load levels with the load ramping rate of $12 \mathrm{MW} / \mathrm{min}$.

To reduce model training sample quantity and shorten training convergence time, half of the original data (taken at every other sampling time) are used to train the model with modified Levenberg-Marquardt algorithm [11]. The training cycle is set for 1000 and the preset Mean Squared Error (MSE) is 1e-6.

\section{Training Results and Analysis}

For the same model structure shown in Fig. 3, different hidden layer nodes are used to train different models. The models' convergence speed and MSE are compared in Table I. It can be seen that, for the simplified NARX model (based on BP neural network) with time-delay inputs and outputs feedback, the model's precision looks not too sensitive to the number of hidden layer neurons, meaning less number of hidden neurons can also achieve high precision. The training results are almost same for taking 12 and 24 hidden layer neurons. With a few training cycle and a shorter training time, the models' MSE can achieve less than 2e-7. After a comprehensive consideration, the number of hidden layer neurons takes 12 .

TABLE I. TRAINING RESUlts OF THE NN MODEL WITH DIFFERENT HIDDEN NODES

\begin{tabular}{c|c|c|c}
\hline $\begin{array}{c}\text { Hidden } \\
\text { Neurons }\end{array}$ & $\begin{array}{c}\text { Training } \\
\text { Time/s }\end{array}$ & $\begin{array}{c}\text { Training } \\
\text { Cycle }\end{array}$ & $\begin{array}{c}\text { Model Error } \\
\text { (MSE) }\end{array}$ \\
\hline 4 & 23.656 & 58 & $9.978 \mathrm{e}-007$ \\
\hline 12 & 10.219 & 12 & $1.9837 \mathrm{e}-007$ \\
\hline 20 & 14.063 & 9 & $9.6841 \mathrm{e}-007$ \\
\hline 24 & 22.672 & 13 & $1.1009 \mathrm{e}-007$ \\
\hline 28 & 37.906 & 18 & $9.9337 \mathrm{e}-007$ \\
\hline
\end{tabular}

The model calculating outputs are compared with the sample data in Fig. 4. It is demonstrated that the model error is very small.

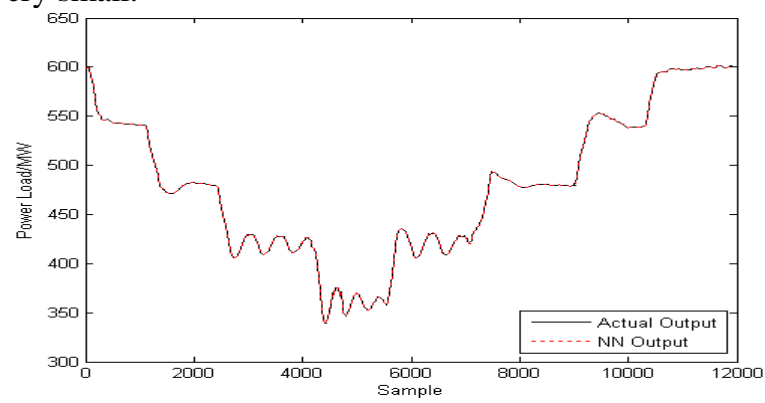

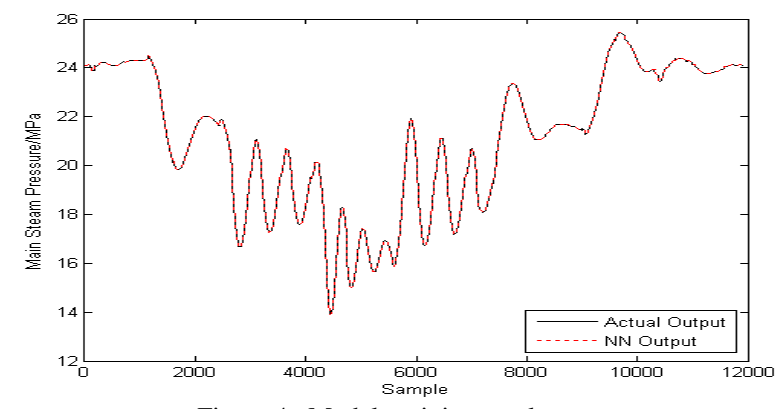

Figure 4. Model training results.

\section{Model VAlidation Tests}

\section{A. Model Validation for Varying-Load Conditions}

To validate the on-line dynamic performance of the trained model, simulation tests under wide-range loadchanging scope are carried out. In loading-down process from 540MW to $360 \mathrm{MW}$ with load changing rate of $10 \mathrm{MW} / \mathrm{min}$, the model outputs for main steam pressure and load are compared with those of actual plant in Fig. 5. It can be seen that model outputs are close to those of plant.
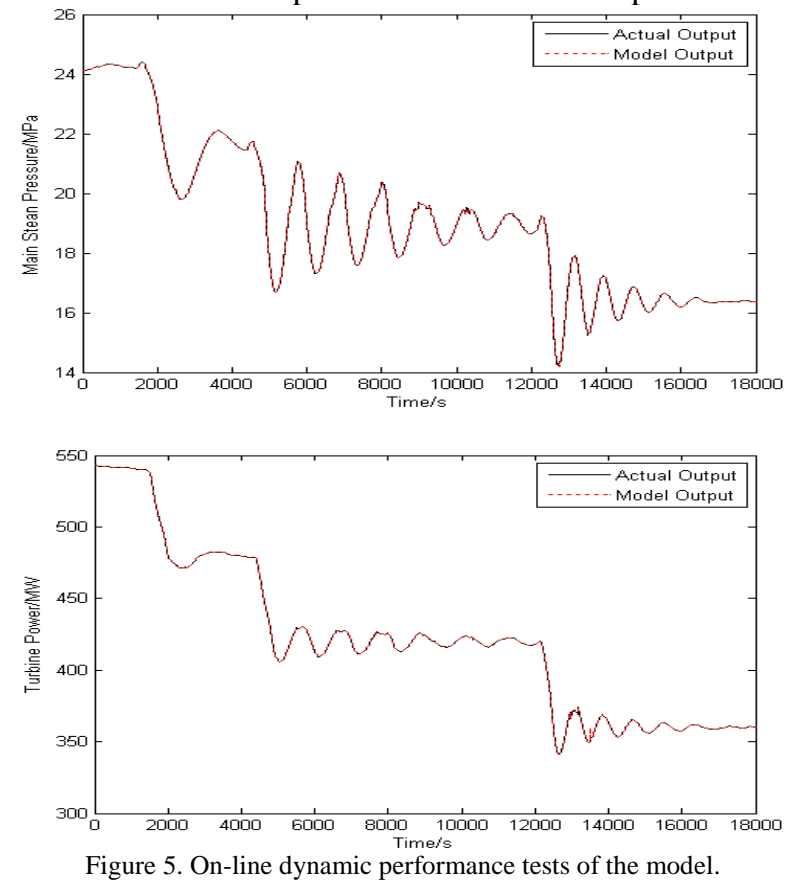

\section{B. Model Step-Disturbance Tests}

On-line experiments are conducted using each input's step disturbance to validate the correctness of the model. The test process is described as follows. The trained model is connected into the simulator at $100 \%$ stable load condition. Then the fuel total operating demand, turbine governing valve opening demand, feedwater demand are cut into manual mode. Respectively, step increase (5\%) only one input value and keep other inputs changeless at the same time, and observe and compare the model outputs and the actual outputs. The test results are shown in Figs. 6 to 8 . 

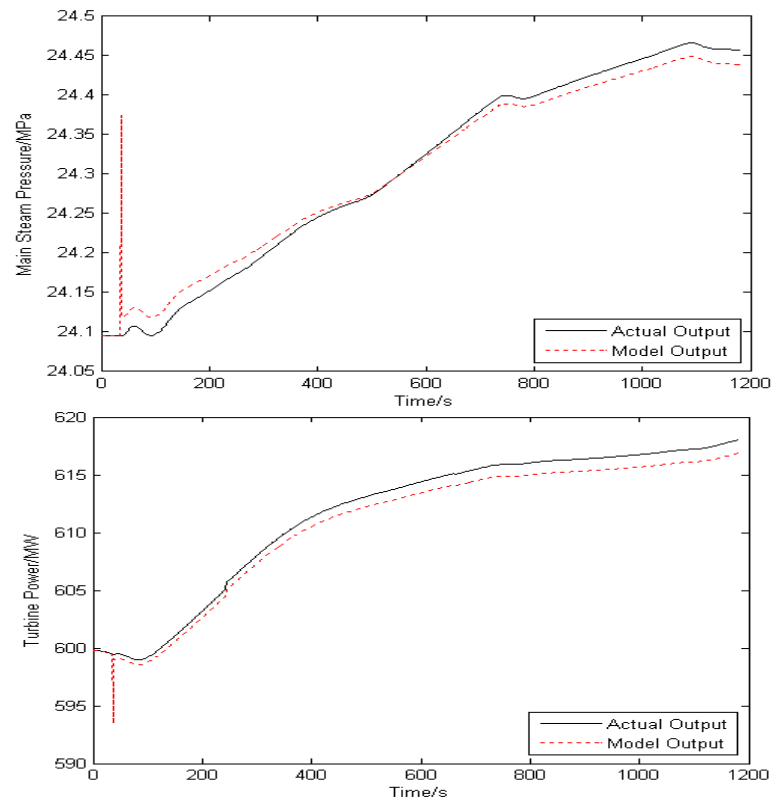

Figure 6. Model response with.fuel instruction step increase 5\%.
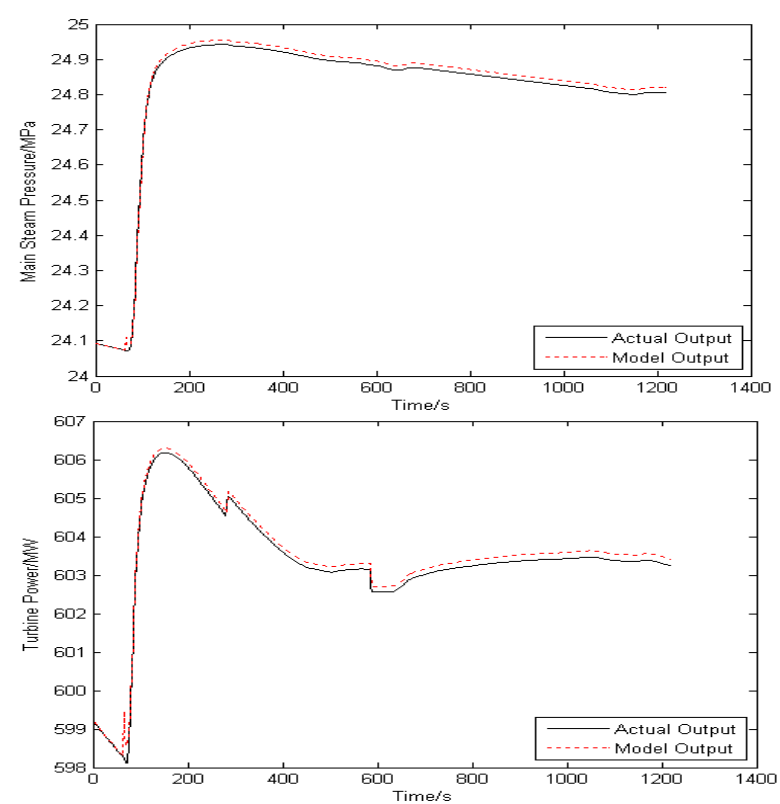

Figure 7. Model response with feedwater instruction step increase $5 \%$.

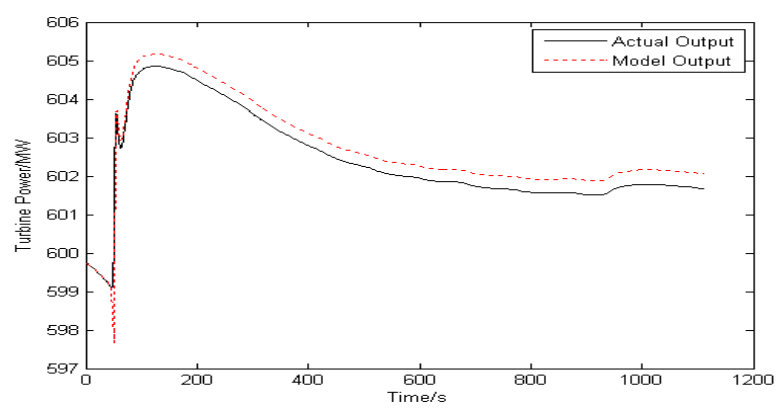

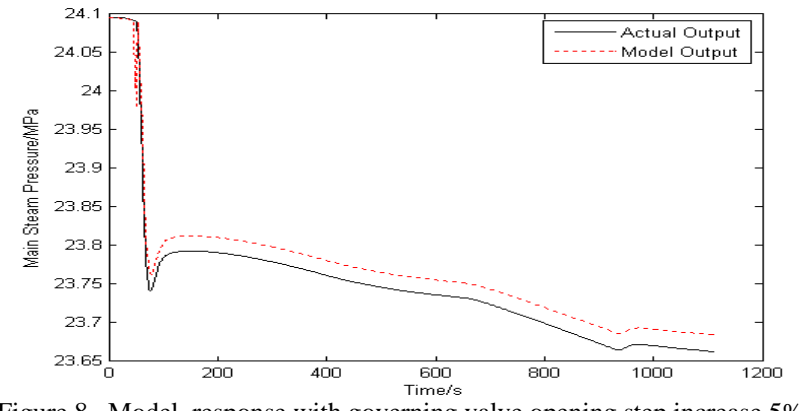

Figure 8. Model response with governing valve opening step increase $5 \%$

It can bee seen from above tests that the response curves of the model and the actual outputs are anastomotic for all the 3 disturbances, which proves that the established neural network model is correct in essence.

\section{CONCLUSION}

In this paper, by taking a $600 \mathrm{MW}$ supercritical coal-fired power generating unit as a modeling object, a NARX model expressed with BP neural network structure was used to build the mathematical model for the unit's load and the steam pressure characteristics. The simulation tests showed that the model can fit the complex non-linear, dynamic characteristics between unit's load, main steam pressure and fuel, feedwater flow and turbine governing valve opening. The model is with high precision and strong generalization ability and can be used as a prediction model for intelligent controller's design of the supercritical boiler unit to meet the engineering application requirements.

\section{ACKNOWLEDGMENT}

This work is supported by the Natural Science Foundation of China under grant 61174111 and the Fundamental Research Funds for the China Central Universities.

\section{REFERENCES}

[1] Z.J. Li, Thermal Automatic Control System, Beijing: China Electric Power Press, 1997.

[2] Y. Liang and J. J. Gu, "Parameter identification for the model of supercritical unit based on genetic algorithm," Electric Power Science and Engineering, Vol. 26, No. 7, pp. 38- 41, Jul. 2010.

[3] J. Ding, G.Y. Li, and N. Wang, "Research on simplified model for the control of 660MW supercritical unit," Electric Power Science and Engineering, Vol. 27, No. 5, pp. 29- 34, May. 2011.

[4] L. Tian, D.L. Zeng, X.P. Liu, and J.Z. Liu, "A simplified non-linear dynamic model of 500MW unit," Power Engineering, Vol. 24, No. 4, pp. 522- 525, Aug. 2004.

[5] S. Yan, D.L. Zeng, J.Z. Liu, and Q.J. Liang, "A simplified non-linear model of a once-through boiler-turbine unit and its application," Proceedings of the CSEE, Vol. 32, No. 11, pp.126- 134, Apr. 2012.

[6] X.S. Tian, Z.X. Han, L.J. Chen, and J. Cai, "Parameter estimation and equilibrium points analysis on non-linear model of the supercritical unit," Computer Simulation, Vol. 28, No. 1, pp. 20- 23, Jan. 2011.

[7] Z.X. Han, C.X. Zhou, W. Zhang, H.J. Ma, C.J. Zhang, and D. Li, "New design method of coordinated control system of supercritical pressure power unit and its application," Proceedings of the CSEE, Vol. 29, No. 8, pp.75- 81, 2009. 
[8] S.Q. Liu, S.F. Yu, and L. Zhou, "Mathematic model and simulation of 900MW supercritical once-through boiler," Computer Simulation, Vol. 22, No. 10, pp. 215- 218, Oct. 2005.

[9] Q.J. Liang, J.Z. Liu, Y.G. Xue, and D.L. Zeng, "A non-linear model for ultra-supercritical power unit and the dynamic characteristic study," Journal of Power Engineering, Vol. 32, No. 2, pp. 118- 123, Feb. 2012.
[10] X. Wang and W.H. Wang, Artificial Neural Network Principle and Application. Shenyang: Northeast University Press, 2000.

[11] H. Demuth, M. Beale, and M. Hagan, Neural Network Toolbox ${ }^{\mathrm{TM}} 6$ User's Guide, The Math Works, Inc. 2008. 